\title{
Changes Of Ascorbic Acid Content In Leaves And Fruits Of Jujube At Different Developmental Stages
}

Jia Guo ${ }^{1, a}$, Nengli Fu²,b, Guoying Song ${ }^{3, c}$, Qunxian Deng ${ }^{4, d *}$

${ }^{1}$ College of Horticulture, Sichuan Agricultural University, Chengdu, China

a2995062401@qq.com, b1530779913@qq.com, c344596038@qq.com, d1324856299@qq.com

${ }^{*}$ Corresponding author.

Keywords: Zizyphus jujuba 'Zhanshanpinguozao'; Leaf; Fruit; Ascorbic Acid

Abstract: It takes Zizyphus jujuba 'Zhanshanpinguozao' as the test material. Changes of Ascorbic Acid content in four stages (young leaves, spreading leaves, mature leaves, senescent leaves) of jujube leaf development, jujube flowers and five periods of fruit development (Young fruit period, green mature period, white mature period, pre-mature period, mature period) were studied. The results showed that the change trends of AsA content in jujube leaves were similar at different stages, which showed that young leaves $>$ spreading leaves $>$ matured leaves $>$ senescent leaves, and DHA content $>$ AsA content after July 14th. While in jujube fruit, AsA content followed the fruit development, which performed first increased and then decreased, and the white mature period was the highest, and the AsA content was always higher than the DHA content. In the same period, the AsA content in the jujube fruit was greater than that of the leaf and the flower of jujube. Therefore, the accumulation rules of Ascorbic Acid in the fruits and leaves of Zizyphus jujuba 'Zhanshanpinguozao' were quite different, and the main forms of Ascorbic Acid in different tissues were different.

\section{Introduction}

Zizyphus jujuba 'Zhanshanpinguozao' is a Zizyphus.Mill plant belonging to Rhanmaceae.R.Br., which is an important economic plant in China. Its fruit is rich in nutrients, such as vitamin C. It is a new excellent fresh variety [1]. L-ascorbic acid (T-AsA) is a water-soluble antioxidant organic molecule widely found in plants. It is also an important antioxidant and enzyme cofactor in plants, and Ascorbic Acid exists mainly in the form of oxidized Ascorbic Acid (AsA) and Dehydroascorbic Acid (DHA) in plants [2-3]. There is a large difference in Ascorbic Acid content among different species. Most fruits contain Ascorbic Acid [4], and Ascorbic Acid content in jujube fruit is much higher than other common fruits [5]. There are also differences in Ascorbic Acid content between different parts of the same variety, such as kiwifruit [6]; and there are also differences in Ascorbic Acid content in the same tissues at different developmental stages, such as sweet cherry [7] and chestnut [8]. At present, there are also some researches on the changes of Ascorbic Acid content in jujube varieties [9], but there are few reports on the research of Ascorbic Acid on the leaves and fruits of Z. jujuba 'Zhanshanpinguozao'. Therefore, this experiment intends to study the changes of Ascorbic Acid content in flower, leaves and fruits of the Z. jujuba 'Zhanshanpinguozao' at different developmental stages to compare the differences in Ascorbic Acid content between different tissues. This study will provide basic data for the study of Ascorbic Acid synthesis and regulation mechanism of jujube, and at the same time provide basic information and reference basis for the efficient use of jujube flowers, leaves and fruits. 


\section{Materials and methods}

Materials. The experiment began in May 2017. The 7-year-old Z. jujuba 'Zhanshanpinguozao' with consistent growth status of plants and consistent field management was selected as the test tree. Plant spacing is $2 \mathrm{~m}$ and row spacing is $3 \mathrm{~m}$, and 3 plants were selected in one plot, repeated 3 times. The experimental site is Zhanshan Village in Santai County.

Experimental Design. Sample of flowers collection: The first and second batch of flowers were collected at the flowering stage. Sample of leaves collection: From the first batch of Falling flowers period when the fruit set, select jujube with consistent growth status for listing. On May 5th, the young leaves were picked, and picked up every 7 days. After that, the mature leaves were picked at the same time. The sample collection began from June 4th and was collected once every 20 days until the fruit matured. The sampling times were June 4th (young fruit period), June 24th (green mature period), July 14th (white mature period), August 3th (color change period), August 23th (matured period). Each sample harvest evenly equal or equal sized fruits equal to or greater than 30 pieces and leaves equal to or greater than 50 pieces, collected at 10:00 am from the plant's east, south, west, north, top and inner halls. Immediately after the harvest, the ice box was used to bring it back to the laboratory, the indicators were repeatedly measured three times.

Use the electronic balance $(0.1 \mathrm{~g})$ to weigh the total weight of the 30 samples, then find the mean value to calculate the single fruit weight; use the digital display vernier caliper to measure the vertical and horizontal diameters of the fruit to draw a curve. The determination of reduced AsA and DHA is based on Kampfenkel's method [10].

\section{Results and Discussion}

Dynamic Changes of Growth and Development of Fruits. The vertical and horizontal diameters and the single fruit weight of Z. jujuba 'Zhanshanpinguozao' fruits increased rapidly from the young fruit period to the white mature period, and then gradually slowed down, indicating that there were two fruit rapid expansion periods (Fig 1). The growth rate of longitudinal and transverse diameters and individual fruit weight of the fruit gradually decreased from the white mature period to the mature period, indicating that this period should be the critical period for the accumulation of organic matter in jujube fruit. In summary, the single fruit weights generally show the trend of "S".

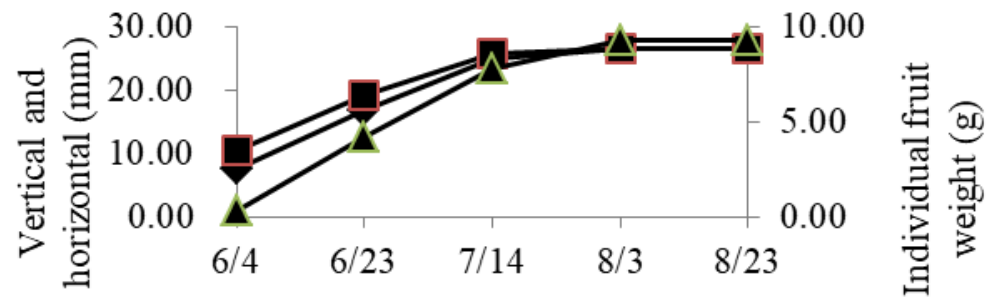

Sample date (month/day)

Fig.1. Growth, development and morphological changes of the fruit of Z. Jujuba Zhanshanpinguozao. Symbols: the transverse diameter (- -$)$, the Longitudinal diameters (- - -), the individual fruit weight (- $\mathbf{\Delta}-)$.

Changes of AsA and DHA Contents in Fruits at Different Development Stages During the entire development process of the fruit of Z. jujuba 'Zhanshanpinguozao', the content of AsA is much higher than that of DHA. The content of AsA began to increase at the young fruit period and peaked at the white mature period. At this time, the AsA content in the fruit was $307.18 \mathrm{mg} / 100 \mathrm{~g} \bullet$ 
FW, which was a $16.7 \%$ increase compared to the young fruit period, and then reduce slowly. The DHA of fruit content was maintained at a low level during the whole fruit development period (Fig 2). It can be seen that the content of AsA is much higher than that of DHA in the fruit, and the content of AsA is highest in the white mature period.

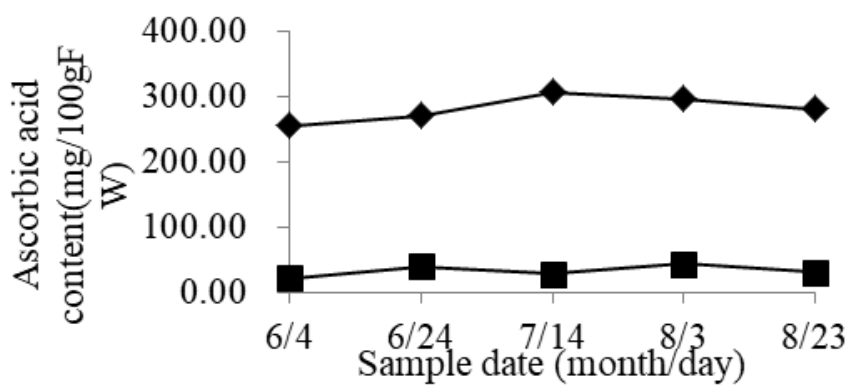

Fig.2. Changes of AsA and DHA content in Z. jujuba 'Zhanshanpinguozao'. Symbols: Ascorbic Acid content(- $\bullet$-), DHA content(-m-).

AsA and DHA Content in Leaves At Different Growth Periods. The content of AsA in the leaves is much larger than that of DHA at the early period of development, but the difference in development is gradually reduced, and the difference between the two levels is not significant around June 24th, after which the DNA content is higher than that of AsA content, there was no difference until August 3th between them. The AsA content continued to decrease throughout the fruit development. The DHA content rapidly decreased from the young leaf to the spreading leaf period (June 4th - July 14th), then began to rise, reached the maximum on July 14th and began to decline during the mature leaf period to the senescent leaf (Fig 3).

Figure 4 shows the proportion of redox state of AsA in the leaf. The overall trend is "first rise, then fall, and then rise." The ratio of ascorbic acid redox status (AsA/DHA) reached the highest in the spreading leaves, began to decline in the mature leaves, and then increased in the senescent leaves. This shows that DHA is closely related to the maturation and aging of the leaves.
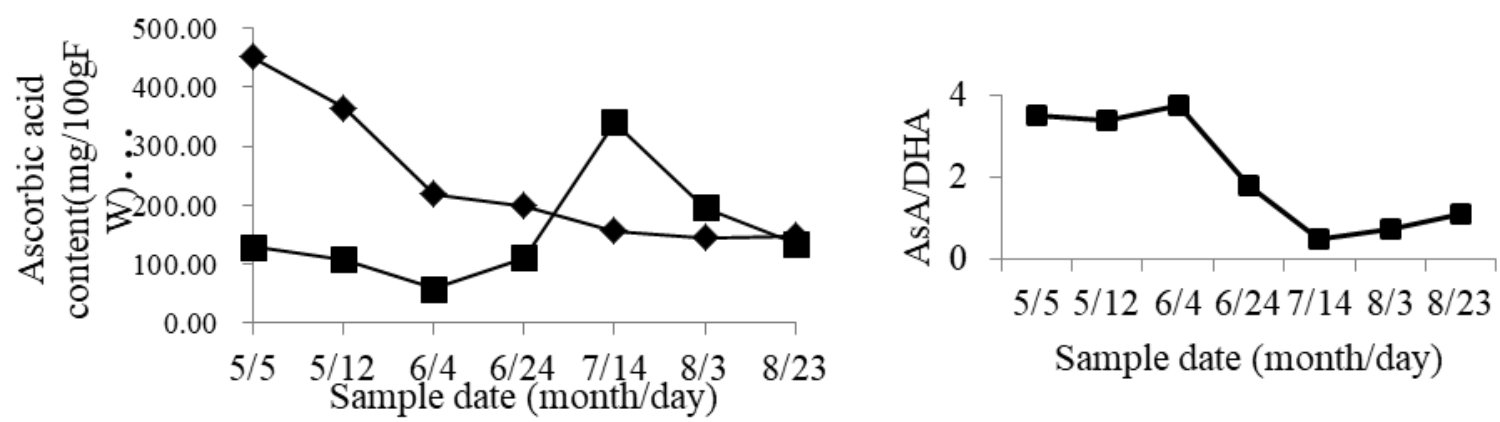

Fig.3. Changes of AsA and DHA content in leaves of Z. jujuba 'Zhanshanpinguozao'. Symbols: Ascorbic acid content(- -), DHA Content(-ш-).Fig.4. Changes of AsA/DHA content in Leaves of $Z$. jujuba 'Zhanshanpinguozao'.

Comparison of Ascorbic Acid Content In The Different Tissues. This experiment compared the contents of Ascorbic Acid in flowers and leaves collected on August 23th. The figure shows that the contents of reduced Ascorbic Acid and oxidized ascorbic acid in jujube fruit, flowers and leaves are quite different. AsA content is fruit $>$ leaf $>$ flower, and DHA content is leaf $>$ flower $>$ fruit (Fig 5).Therefore, the existence of Ascorbic Acid in fruits, flowers and leaves is quite different, and the total amount is also different. 


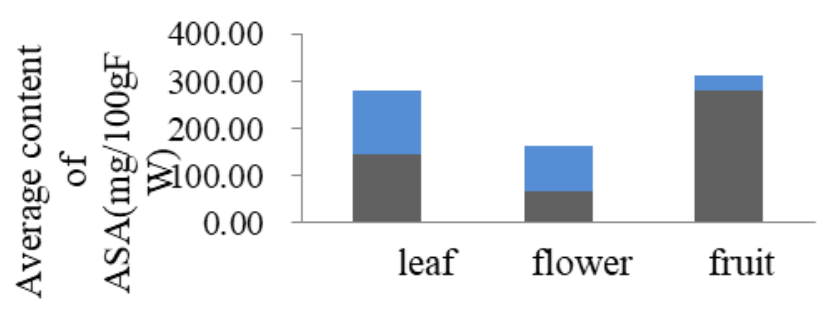

Fig.5.Comparison of AsA and DHA contents indifferent parts of jujube. Symbols: $\square$ (DHA content), (Ascorbic acid content)

\section{Conclusions}

This experiment found that the AsA content in the leaves and fruits of Z. jujuba 'Zhanshanpinguozao' in different periods was very different. AsA content was highest in young leaves and lowest in senescent leaves; DHA content was highest in mature leaves. The content of AsA in fruits increased rapidly between the green mature period and the white mature period, and reached the maximum during the white mature stage. The content of DHA in fruits was always at a low level. In the same period, the content of AsA in leaves, fruits and flowers of jujube differs greatly. The content of DHA in leaves and flowers is much larger than that of fruits, while the content of AsA in fruits is much larger than that of leaves and flowers. Therefore, there are differences in the accumulation of Ascorbic Acid in different tissues, and there are also great differences in the main forms of Ascorbic Acid in different tissues at the same time.

\section{Acknowledgements}

This work was financially supported by the Double subject construction plan of Sichuan Agricultura 1 University and Special action plan projectof science and technology rich people and strong county of Sichuan Province "Integration and demonstration of key Technologies for industrialized develop ment of Z. jujuba 'Zhanshanpinguozao' of Santai County".

\section{References}

[1] Gallie D R. L-ascorbic Acid: Scientifica Vol. 2013 (2013), p. 79.

[2] L. Wang, A. M. Min, Z. G. Tan. Western China College of Agriculture and Forestry Vol. 43(2014), p. 48.

[3] L. He, J. Lu, X.L. Mao, W.H. Chen. Hubei Agricultural Sciences Vol. 52 (2013), p. 1420.

[4] S.W. Yang. Anhui Agricultural Science Bulletin Vol. 17 (2011), p. 34.

[5] S.J. Gong, Z.H. Zhao, M. J. Liu. Journal of Food Agriculture \& Environment Vol. 12 (2014), p. 169.

[6] J.L. Tang, W.U. Han, B.B. Lang. Acta Horticulturae Sinica Vol, 6(2014), p. 1198.

[7] D. Liang, T. Zhu, Z. Ni. Plos One Vol. 12 (2017), p. 14.

[8] M. Hang, Q. Xua, X. X. Deng. Journal of Plant Physiology Vol. 171 (2014), p. 1205.

[9] Y.Y. Chen. Mechanism of Ascorbic Acid Metabolism in sour Jujube and Jujube Fruits (thesis of master degree, Hebei Agricultural University, Tianjin, China 2015).

[10] K. Kampfenkel, M. V. Montagu, Analytical Biochemistry Vol. 225 (1995), p. 165. 